\title{
Ciência e Tecnologia em um programa de infotainment: uma análise de conteúdo da cobertura do Fantástico*
}

\author{
Flavia Natércia da Silva Medeiros** \\ Marina Ramalho*** \\ Cristina Caldas***** \\ Luisa Massarani******
}

\section{Resumo}

Apesar do espaço crescente que a Ciência e a Tecnologia (C\&T) têm ocupado na grade televisiva, poucos estudos se dedicam a examinar como programas de

* Este estudo contou com apoio do Conselho Nacional de Desenvolvimento Científico e Tecnológico (CNPq), da Fundação Carlos Chagas Filho de Amparo à Pesquisa do Estado do Rio de Janeiro (Faperj) e do Programa Iberoamericano de Ciencia y Tecnología para el Desarrollo (Cyted).

** Colaboradora do Labjor, Universidade de Campinas (Unicamp), Campinas-SP, Brasil. Pós-doutorado em Divulgação e Percepção Pública da Ciência no Laboratório de Estudos Avançados em Jornalismo da Unicamp, doutorado em Comunicação Social pela Universidade Metodista de São Paulo e mestrado em Ecologia e graduação em Ciências Biológicas na Universidade Estadual de Campinas.E-mail: fnatercia@yahoo.com

*** Jornalista e pesquisadora do Núcleo de Estudos da Divulgação Científica do Museu da Vida, Casa de Oswaldo Cruz, Fiocruz, Rio de Janeiro-RJ, Brasil. Doutoranda em Educação, Gestão e Difusão em BioCiências na Universidade Federal do Rio de Janeiro. Possui máster em Jornalismo pela Universidade Rey Juan Carlos, Espanha. E-mail: marinar@fiocruz.br

**** Coordenadora do Setor de Cooperação Educacional no Consulado-Geral do Brasil em Boston, Massachusetts, Estados Unidos. Jornalista freelancer. Doutora em Imunologia pela Universidade de São Paulo, mestre em Biologia Molecular pela Universidade de Brasília e especializada em Jornalismo Científico pelo Labjor-Unicamp. E-mail: caldas.cr@gmail.com

***** Pesquisadora do Núcleo de Estudos da Divulgação Científica do Museu da Vida, Casa de Oswaldo Cruz, Fiocruz, Rio de Janeiro- RJ, Brasil. É Honorary Research Associate na University College London, Reino Unido. Bolsista Produtividade do CNPq 1D. Doutora pela Universidade Federal do Rio de Janeiro. E-mail: lumassa@fiocruz.br 
infotainment abordam esses temas. O objetivo deste estudo é analisar a cobertura de C\&T realizada pelo programa dominical Fantástico, da Rede Globo, de modo a identificar suas principais características e o espaço ocupado por estes temas na agenda do programa. Foi utilizada a metodologia de análise de conteúdo. Verificamos, entre outros aspectos, que a área de conhecimento abordada com maior frequência é a de Medicina \& Saúde e que predominaram os frames "nova pesquisa" e "personalização". Cientistas e médicos, além de cidadãos comuns, foram as fontes mais consultadas, sugerindo que Fantástico busca conciliar a construção de credibilidade e a identificação com as audiências.

Palavras chave: Televisão. Infotainment. Ciência e Tecnologia. Análise de conteúdo.

\section{Science and Technology in an infotainment program: a content analysis of Fantástico's coverage}

\section{Abstract}

In spite of the increasing space Science and Technology (S\&T) have been occupying in television, few studies have been done on the way infotainment programs approach these issues. The goal of this study is to analyze the S\&T coverage held by Fantástico, a Sunday program from Rede Globo, to identify their main features and the space occupied by these issues on the program agenda. The methodology used was content analysis. We observed, among other aspects, that the most present knowledge area was Medicine \& Health and that "new research" and "personalization" were the most frequent frames. Scientists and physicians, as well as average citizens, were the main sources, suggesting that Fantástico tries to conciliate the building of credibility and the identification with the audiences.

Keywords: Television. Infotainment. Science and Technology. Content analysis.

\section{Ciencia y Tecnología en un programa de infotainment: un análisis de contenido de la cobertura de Fantástico}

\section{Resumen}

A pesar del espacio creciente que la Ciencia y la Tecnología (CyT) ocupan en la programación televisiva, pocos estudios han investigado cómo dichos temas son abordados en programas de infotainment. El objetivo de este estudio es analizar la cobertura de CyT realizada por el programa dominical Fantástico, de Rede Globo, para identificar sus principales características y el espacio ocupado por estos temas en la agenda del programa. La metodología utilizada fue la de análisis de contenido. Verificamos, entre otros aspectos, que el área de conocimiento abordado con mayor frecuencia fue Medicina y Salud, y que fueron más frecuentes los frames "nueva investigación" y "personalización". Los científicos y los médicos, además de los ciudadanos comunes, fueron las fuentes más consultadas, lo que sugiere que Fantástico busca conciliar la construcción de credibilidad y la identificación con las audiencias.

Palabras clave: Televisión. Infotainment. Ciencia y Tecnología. Análisis de contenido. 


\section{Introdução}

Ciência e a Tecnologia têm assumido cada vez mais um
papel importante na vida dos cidadãos, incluindo (sem
ficar limitado a) os avanços que contribuem para a melhoria da qualidade de vida, como o desenvolvimento de vacinas e medicamentos, até a melhor compreensão do mundo que nos rodeia e a intervenção sobre ele, por exemplo, dando mais subsídios para a mitigação das mudanças climáticas. A televisão é a principal fonte de informação sobre Ciência e Tecnologia (C\&T) para os cidadãos de diversos países (MILLER, AUGENBRAUN, SCHULHOF et al., 2006; NISBET, SCHEUFELE, SHANAHAN et al., 2002). No entanto, é comum que temas relacionados com C\&T ocupem um espaço marginal nos programas noticiosos televisivos, menor que o dedicado à política ou aos esportes.

Analisando os principais programas noticiosos do horário nobre de canais públicos e privados dos maiores mercados da Europa, León (2008) observou que apenas 1,7\% do número total de matérias transmitidas tratavam de C\&T, durando em média 60,7 segundos. Matérias sobre Medicina e Saúde e sobre Meio Ambiente representaram 4,5\% e 2,0\% do total, respectivamente. Considerando esses três assuntos relacionados com Ciência, as matérias representaram $8,2 \%$, valor menor que o obtido para Política (16,5\%), Esportes (13,1\%) e Crime (9,7\%) (LEÓN, 2008). Em sua análise de seis telejornais públicos europeus, Verhoeven (2010) observou que uma média de 5,7\% do tempo dos programas foi dedicada a C\&T e que as matérias sobre temas de Ciência duraram em média 85,4 segundos. No Brasil, um estudo mostrou um valor um pouco superior no Jornal Nacional: 7,3\% da cobertura se referiram a temas de C\&T, com uma duração média das matérias de 135 segundos (RAMALHO, POLINO, MASSARANI, 2012).

Mais do que em programas informativos, porém, Ciência e Tecnologia são assuntos frequentes em filmes, séries, documentários e outros programas de entretenimento (VERHOEVEN, 2010; DINGWALL, ALDRIDGE, 2006; WEINGART, MUHL, PANSE- 
GRAU, 2003; ALDRIDGE, DINGWALL, 2003). Uma medida do efeito que a exposição a esse tipo de programa pode surtir vem do estudo de Besley e Shanahan (2005), que observaram que pessoas que afirmaram assistir mais a programas de entretenimento e de Ciência na televisão manifestaram com maior frequência sentimentos positivos quanto às Biotecnologias. No que concerne à preocupação com questões ambientais, Holbert, Kwak e Shah (2003) constataram que tanto os telejornais, que costumam tratar desses temas quando ocorre algum problema - gerando coberturas dramáticas e episódicas -, quanto os programas de atualidades e de natureza, que transmitem mensagens predominantemente positivas, contribuíram para que os telespectadores se declarassem mais dispostos a reciclar seu lixo ou reduzir o consumo de energia.

Da mesma forma, o filme $\mathrm{O}$ dia depois de amanhã, por exemplo, apesar dos exageros com que trata o tema do aquecimento global, surtiu efeitos positivos sobre o público de Norwich, no Reino Unido: contribuiu para aumentar a consCiência e a compreensão sobre as mudanças climáticas, bem como motivou mudanças de atitude (LOWE, BROWN, DESSAI et al., 2006). Em um estudo com grupos focais no estado da Geórgia, nos Estados Unidos, ficou evidente que os documentários televisionados sobre questões sociais, históricas e políticas contribuíram para a construção das percepções do público sobre a genética (BATES, 2005). Apesar de o entretenimento não ter, necessariamente, compromisso com a verossimilhança (JÖRG, 2003), programas como os documentários e as dramatizações da emissora britânica BBC gozam de alta credibilidade e legitimidade e servem de parâmetro de qualidade para o público (LOWE, BROWN, DESSAI et al., 2006).

Entre os programas noticiosos/informativos e os de entretenimento, encontram-se os de infotainment, que procuram ao mesmo tempo informar e entreter e têm ocupado um espaço crescente na grade televisiva. Alguns autores alertam, no entanto, que pode haver certa tensão entre o entretenimento e a narração dos fatos (VETTEHEN, NUIJTEN, BEENTJES, 2006; SURETTE, OTTO, 2002; SIQUEIRA, 1998). Se a ênfase é colocada no entretenimento, pode ser, em alguma medida, negligenciada a preocupação 
com a apresentação dos conteúdos - pelo menos na forma de apresentação da informação tal como se espera dos programas noticiosos - (GÖRKE, RUHRMANN, 2003). Nos Estados Unidos, observou-se que os programas de infotainment sobre crime e justiça exageram a prevalência de crimes violentos, super-representam a proporção de crimes efetivamente solucionados e tendem a reforçar estereótipos como o de que criminosos são inerentemente diferentes dos cidadãos que obedecem às leis (SURETTE, OTTO, 2002).

No entanto, são ainda escassos os estudos que se dedicam a analisar os programas de infotainment, especialmente no que se refere à Ciência e Tecnologia, temas de interesse deste artigo. No Brasil, alguns autores se dedicaram a analisar a cobertura de Ciência de programas desse tipo, como Siqueira (1998) e Rondelli (2004), mas claramente há uma demanda por mais estudos, visando ter resultados mais sistematizados, aprofundados e atualizados. Buscando caminhar neste sentido, nosso estudo apresenta uma análise de conteúdo da cobertura de Ciência e Tecnologia pelo Fantástico, programa brasileiro dominical de infotainment.

\section{Metodologia}

Neste estudo, realizamos uma análise de conteúdo do programa Fantástico, atração dominical transmitida pela TV Globo às 20h45min. Criado em 1973, o programa se propõe a informar, entreter e emocionar suas audiências. Como uma "revista eletrônica de variedades", apresenta notícias, reportagens e quadros fixos, voltados a temas específicos e presentes em todas as edições. Escolhemos este programa por ser um dos líderes de audiência em horário nobre (PADIGLIONE, 30 dez. 2011), assistido por uma média de um milhão de pessoas somente na Grande São Paulo todo domingo. Além disso, trata-se de um programa que, embora não se dedique especialmente à divulgação científica, trata regularmente de temas científicos (SIQUEIRA, 1999).

As edições do programa Fantástico veiculadas pelo período de um ano foram gravadas em DVD e assistidas na íntegra. Nossa análise foi feita por um período de 12 meses (abril de 2009 a março 
de 2010), incluindo a quase totalidade dos programas exibidos no período, somando 51 edições ${ }^{1}$. Um aspecto fundamental de nossa metodologia se refere ao processo de definição das matérias que deveriam entrar na amostra. Isso porque, enquanto estudos que se dedicam a editorias de Ciência de jornais impressos têm um escopo claramente definido, em nosso caso não há uma definição editorial a priori do que deve ser considerado como matéria de Ciência e Tecnologia. A consolidação da amostra é, portanto, chave para a validade do próprio estudo e a compreensão de seus resultados.

Por isso, construímos um protocolo de inclusão/exclusão, consolidado por pesquisadores integrantes da Rede Ibero-Americana de Monitoramento e Capacitação em Jornalismo Científico ${ }^{2}$, que teve como ponto de partida a proposta de Rondelli (2004). Para configurar como matéria de Ciência e ser incluída na amostra para análise, a matéria deveria atender, pelo menos, a um dos seguintes requisitos: mencionar cientistas, pesquisadores, professores universitários ou especialistas em geral (desde que aparecessem vinculados a uma instituição científica e comentassem temas relacionados com Ciência) ou mencionar instituições de pesquisa e universidades; mencionar dados científicos ou resultados de investigações; mencionar política científica; tratar de divulgação científica.

Uma vez identificadas as matérias de Ciência, estas foram submetidas a uma análise de conteúdo com base em protocolo também desenvolvido no âmbito da Rede Ibero-Americana anteriormente mencionada. Tal protocolo visou analisar as matérias de Ciência usando diversas variáveis, dentre as quais selecionamos para este artigo as seguintes: duração; presença na chamada de abertura do programa; bloco do telejornal em que foram veiculadas; fazer ou não parte de séries de reportagens sobre Ciência; áreas de conhecimento abordadas; frames (enquadramentos) utilizados; fontes

\footnotetext{
${ }^{1}$ Apenas um programa não foi analisado, por problemas técnicos.

2 A Rede Ibero-americana de Monitoramento e Capacitação em Jornalismo Científico foi criada em 2009, com apoio do Programa Iberoamericano de Ciencia y Tecnología para el Desarrollo Científico (Cyted). Coordenada pelo Museu da Vida, reúne instituições de dez países. Para mais informações, ver www.museudavida.fiocruz.br/redejc
} 
utilizadas; uso de recursos visuais (animação, tabelas, infográficos e esquemas), presença de imagens de cientistas; local onde foram mostrados os cientistas; gênero dos cientistas entrevistados; países de origem do evento noticiado; menção a benefícios e danos da Ciência; presença/ausência de controvérsias e de explicações de termos ou conceitos científicos.

Mais detalhadamente: em cada matéria incluída na análise começamos por registrar o destaque relativo - avaliado por meio de sua presença ou não na chamada de abertura do programa, do bloco do programa em que apareceu, da inserção em séries de reportagens e da duração - que recebeu. $\mathrm{O}$ tempo disponível para contar uma história representa um recurso limitado e disputado na televisão (NUCCI, KUBEY, 2007). A duração pode ser encarada como um indicador da importância que o programa atribui a cada tópico e pode ter relação com a qualidade - matérias mais longas teriam, por exemplo, mais espaço para contextualização e explicações científicas (LEÓN, 2008; ALDRIDGE, DINGWALL, 2003).

A principal área do conhecimento de que trataram as matérias foi classificada nas seguintes categorias: Ciências Exatas e da Terra; Engenharias e Tecnologias; Ciências Agrárias; Ciências Biológicas; Ciências Ambientais; Medicina e Saúde; Ciências Sociais e Humanidades; Ciência e Tecnologia como um todo; outra. Já os frames (enfoques atribuídos às notícias) principais considerados neste estudo foram os seguintes: nova pesquisa; novo desenvolvimento tecnológico; background científico; impacto da Ciência e da Tecnologia; ética/moral; estratégia política, políticas públicas ou regulação; mercado, promessa econômica, patentes e direito de propriedade; controvérsia científica; incerteza científica; personalização; cultura; nenhum dos anteriores; outro. Registramos a presença de até três frames por matéria. Isso foi feito porque, ao testar em que medida codificadores distintos convergiam/divergiam em sua resposta, observamos que o valor de divergência era alto quando solicitávamos que escolhessem um único frame, mas era reduzido quando solicitávamos que escolhessem três. Em outras palavras, havia certo consenso sobre os principais frames; as diferenças se davam apenas no momento de hierarquizá-los. 
Também registramos se houve menção a benefícios, promessas, danos e riscos da Ciência e a controvérsias - distinguindo entre controvérsias de natureza científica e de outra natureza - e se havia explicações de termos científicos. A explicação de termos deve facilitar a compreensão dos assuntos abordados pelos telespectadores, no entanto, costuma ser pouco frequente na cobertura da Ciência pela mídia (LEÓN, 2008). Quanto ao uso de recursos visuais, registramos a presença de animação ou tabelas, infográficos e esquemas; eles são usados para ilustrar, explicar, sumarizar, destacar e especular. Observamos ainda se as matérias mostraram imagens de cientistas e o gênero dos cientistas entrevistados.

A origem geográfica do evento noticiado foi classificada nas categorias que seguem: Brasil, América Latina (menos Brasil), América do Norte, Europa, outros países desenvolvidos, outros países em desenvolvimento e diversos países e continentes. Já os lugares onde apareceram os cientistas foram classificados em: laboratório, escritório, hospital/consultório médico, coletiva de imprensa, locais onde realizam pesquisa de campo, casa de família, espaço público (jardim/parque/praça/rua), estúdio e outros.

\section{Resultados}

Identificamos 111 matérias ao longo dos 12 meses analisados, ou seja, em média um pouco mais de duas matérias sobre temas de Ciência e Tecnologia por programa. As matérias duraram em média 346 segundos; a mais curta durou 21 segundos e a mais longa teve 1783 segundos (29 minutos e 43 segundos). A mediana da distribuição foi 301 segundos ( $\sim 5$ minutos), ou seja, metade das matérias durou mais que este valor, metade menos. Foram feitas chamadas na abertura do programa para mais da metade das matérias analisadas (55\% do total). Uma proporção de $16,2 \%$ das matérias foi exibida no primeiro bloco do programa e porcentagem idêntica $(16,2 \%)$ integrou séries de reportagens.

Três áreas do conhecimento - Medicina e Saúde (41,4\%), Ciências Biológicas (15,3\%) e Ciências Sociais/Humanidades (15,3\%) - tiveram frequência expressiva, conforme mostra a figura 1. 
Figura 1 - Principal área de conhecimento das matérias.

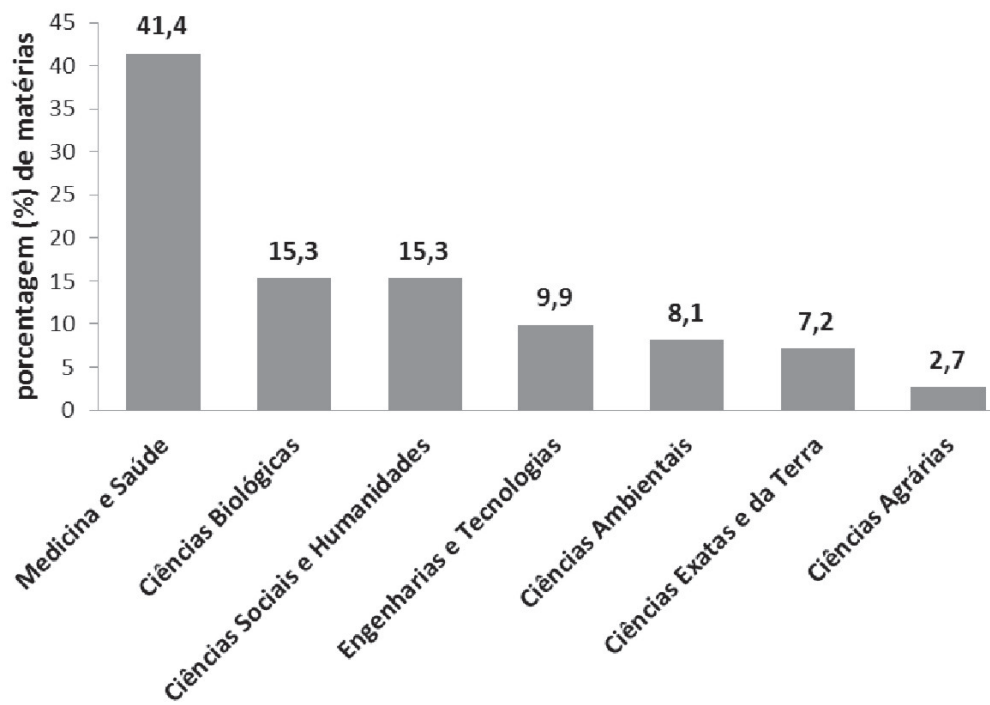

Legenda: proporção de matérias por área de conhecimento.

A duração média das matérias segundo áreas de conhecimento foi assim distribuída: Medicina e Saúde (412 segundos), Ciências Ambientais (390 segundos), Ciências Exatas e da Terra (341 segundos), Engenharias e Tecnologias (337 segundos), Ciências Sociais e Humanidades (298 segundos), Ciências Biológicas (250 segundos) e, por fim, Ciências Agrárias (78 segundos).

Medicina e Saúde foram também os temas mais presentes nas matérias que mereceram chamada na abertura do programa $(50,8 \%)$ e os mais frequentes entre as matérias mais longas. Foram maioria também nas matérias exibidas no primeiro bloco do programa (44,4\%). Dentre as 93 matérias exibidas nos blocos intermediários, 40,9\% trataram de Medicina/Saúde. Nenhuma matéria de Ciência em geral foi observada no último bloco, espaço geralmente dedicado ao futebol. Por sua vez, dentre os $16,2 \%$ de matérias que integraram uma série, predominaram como área de conhecimento principal: Ciências Biológicas (33,3\%), Ciências Ambientais (27,8\%) e Medicina/Saúde (27,8\%). 
Da lista inicial de dez frames, constatamos que apenas dois deles estiveram presentes em um maior número de matérias: (1) novos resultados de pesquisa, quando o enfoque da matéria recaía sobre o anúncio de uma descoberta, de novos medicamentos ou tratamentos; e (2) personalização, quando o enfoque estava na experiência ou testemunho de uma pessoa, como por exemplo, o de pacientes submetidos a um tipo específico de tratamento. A figura 2 mostra a distribuição de frames.

Figura 2 - Distribuição de matérias de CËT por frame.

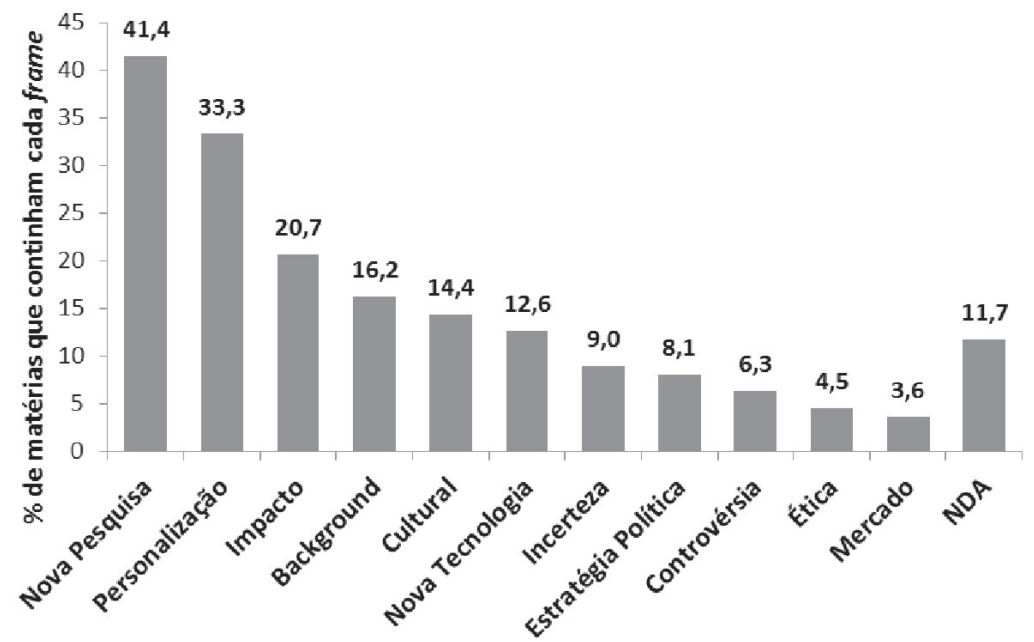

Legenda: proporção (\%) de matérias que apresentaram cada um dos frames.

As fontes citadas com mais frequência foram os cientistas (em $96,4 \%$ das matérias) e os cidadãos comuns $(78,4 \%)$, seguidos por médicos $(34,2 \%)$, outros especialistas $(31,5 \%)$ e membros do governo $(18,9 \%)$. Outras fontes foram menos expressivas.

programa deu preferência a eventos do próprio país: estudos sobre temas brasileiros foram objeto de 68 matérias (61,2\% do total), enquanto que pesquisas sobre temas da América do Norte estiveram presentes em 33 matérias (29,7\% das matérias), seguidas por estudos em torno de temas europeus - 13 matérias (11,7\%). Estudos envolvendo temas latino-americanos foram inexpressivos, como mostra a figura 3: 
Figura 3 - Número de matérias de CËT por país de origem do evento.

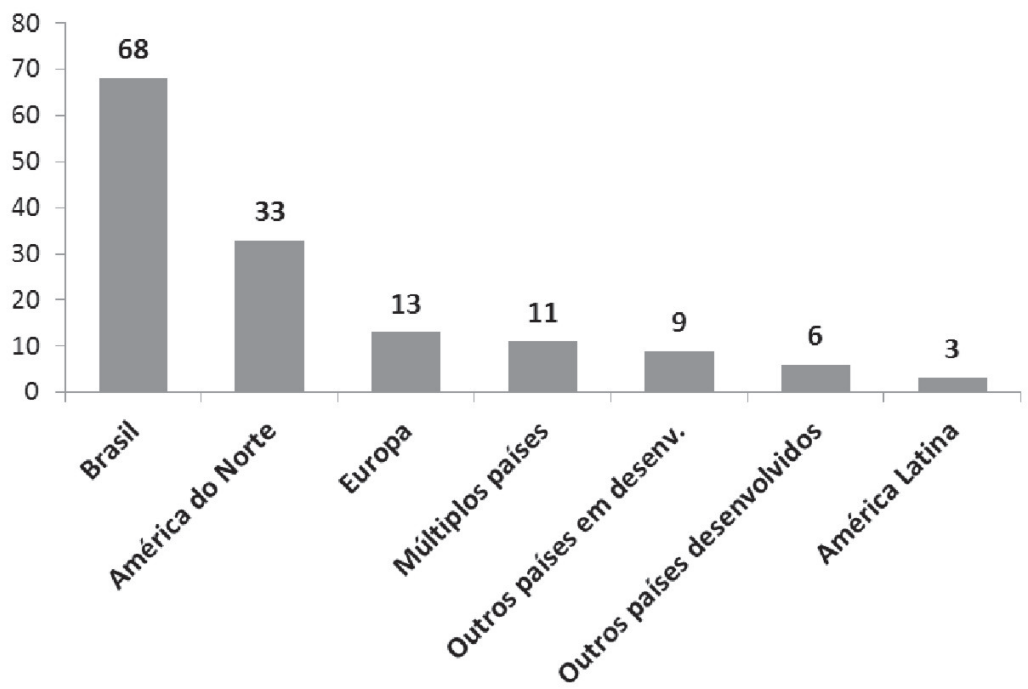

Legenda: número absoluto de matérias de Ciência e Tecnologia por país de origem do evento noticiado. A soma das matérias supera 111 porque uma mesma matéria poderia citar mais de um país de origem.

Animações como recursos visuais foram usadas em $73 \%$ das matérias. Também foi frequente o uso de tabelas, infográficos e esquemas, que apareceram em $64 \%$ das matérias. Animações foram utilizadas com maior frequência em matérias sobre Medicina e Saúde (43,2\%), Ciências Biológicas (17,3\%) e Ciências Sociais/Humanidades $(13,6 \%)$. Tabelas, infográficos e esquemas, por sua vez, foram mais usados em matérias sobre Medicina e Saúde (45,1\%); Ciências Sociais/Humanidades (16,9\%), Ciências Biológicas (15,5\%) e Ciências Ambientais (12,7\%). Em 60,4\% das matérias, houve explicação de termos científicos. Explicações científicas foram mais frequentes nas matérias sobre Medicina/Saúde (49,3\%), Ciências Biológicas (20,9\%) e Ciências Exatas e da Terra (10,5\%). Imagens de cientistas, por sua vez, apareceram em 79,3\% das matérias. Cientistas do sexo masculino foram entrevistados em $58,6 \%$ das matérias e cientistas mulheres, em $28 \%$ do total. Cientistas foram mostrados, sobretudo, no laboratório, no escritório e no hospital, como mostra a figura 4: 
Figura 4 - Lugares onde aparecem os cientistas.

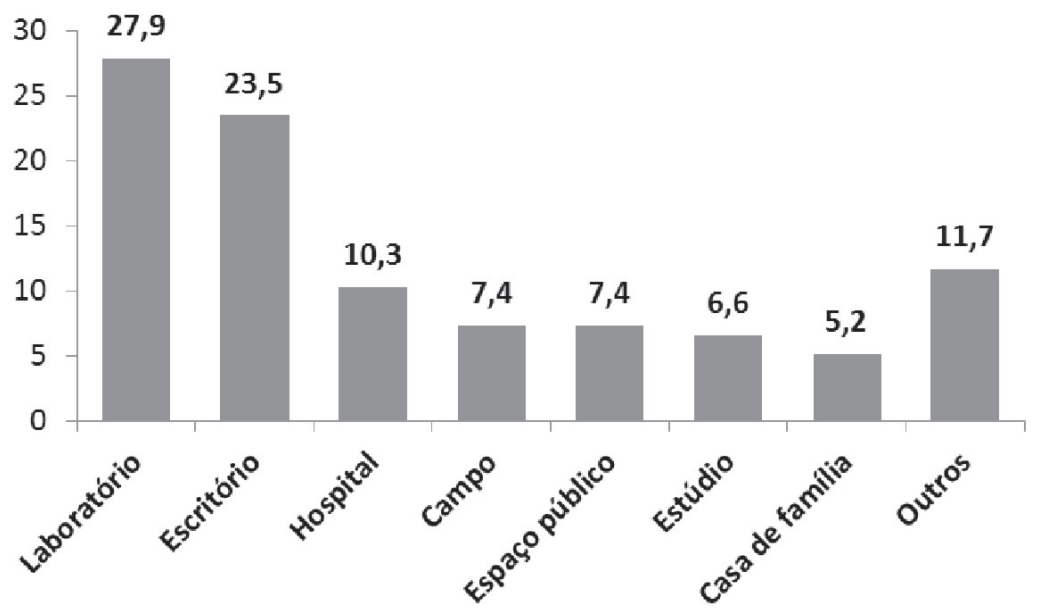

Legenda: frequência relativa dos lugares nos quais foram mostrados os cientistas nas matérias do Fantástico.

Benefícios concretos $(20,7 \%)$ da Ciência e suas promessas para o futuro $(26,1 \%)$ foram mais frequentes nas matérias do que riscos $(15,3 \%)$ e danos $(8,1 \%)$ da Ciência. Aspectos positivos foram, portanto, mencionados com maior frequência que os negativos. Controvérsias foram mencionadas em 37 matérias, um terço do total. Dentre estas, controvérsias científicas representaram $29,7 \%$ do total, enquanto controvérsias de outra natureza representaram $70,3 \%$.

\section{Discussão}

Conforme observaram Siqueira (1998) e Rondelli (2004), constatamos que Ciência e Tecnologia, abordadas desde a primeira edição do Fantástico em 1973, continuam tendo espaço reservado no programa. Além de frequentes, temas de C\&T receberam destaque no dominical da TV Globo: um percentual relevante de matérias foi objeto de chamadas de abertura. $\mathrm{O}$ destaque pode estimular telespectadores a assistir à reportagem e a se interessar 
pelo assunto em questão. Ramalho, Polino e Massarani (2012), por sua vez, observaram que a C\&T faz parte da agenda do Jornal Nacional, outro programa da mesma emissora que é líder de audiência em horário nobre. Tendo esses programas um papel na consolidação da agenda jornalística no Brasil, os resultados sugerem que uma parte importante da mídia brasileira considera temas de C\&T relevantes para a sociedade - sem colocar em discussão, aqui, a qualidade dos produtos midiáticos veiculados.

Por outro lado, foi relativamente baixa a proporção de matérias relacionadas com Ciência e Tecnologia apresentadas em séries. A inserção de uma reportagem em uma série pode aumentar as chances de atrair a atenção do público para determinados temas e convidar as audiências a refletir sobre os mesmos, o que, em alguma medida, pode levar a mudanças de percepções ou de atitudes. Um experimento sobre os efeitos de um programa educacional verificou que uma série de programas sobre a questão das drogas, transmitida por três noites consecutivas pela rede norte-americana de televisão PBS produziu efeitos duradouros sobre as pessoas que participaram do estudo, refletindo a mensagem principal do programa: no lugar do encarceramento, passaram a defender a necessidade de investimento público em centros de reabilitação e a diversificação das opções de tratamento para os usuários de drogas. No que se refere ao conhecimento sobre o assunto, porém, o programa não surtiu efeito (ALBERTSON, LAWRENCE, 2009).

As matérias de Ciência veiculadas no Fantástico, por conta do próprio perfil do programa, foram em média mais longas do que a média observada em programas noticiosos por diversos estudos. Em telejornais e programas de atualidades da televisão australiana, Chapman, Holding e Ellerm et al. (2009) verificaram que as matérias sobre Saúde/Medicina veiculadas ao longo de 47 meses, entre maio de 2005 e março de 2009, duraram em média 97 segundos. No Brasil, as matérias do Jornal Nacional têm duração média de 135 segundos (RAMALHO, POLINO, MASSARANI, 2012).

Seguindo uma tendência internacional (DURANT, EVANS, THOMAS, 1992; BUCCHI, MAZZOLINI, 2003, LEÓN, 2008), matérias sobre temas de Medicina e Saúde foram mais frequentes 
que outros temas no Fantástico. Nessas matérias, foram usados com maior frequência recursos visuais como animações, tabelas, gráficos e esquemas explicativos. E, ainda, essas matérias receberam maior destaque e foram mais longas, o que também foi observado em outros estudos sobre a cobertura de C\&T pela mídia. No Jornal Nacional, Medicina e Saúde foram também os temas de destaque na cobertura de Ciência (RAMALHO, POLINO, MASSARANI, 2012). Estudos realizados em países como Reino Unido, Canadá e Itália (DURANT, EVANS, THOMAS, 1992; EINSIEDEL, 1992, BUCCHI, MAZZOLINI, 2003) sugerem que a Medicina é a principal referência para o imaginário social em relação à Ciência, representando um paradigma da Ciência em geral. Além disso, o público manifesta grande interesse por temas de Medicina \& Saúde, que são vistos pelos jornalistas como sendo mais passíveis de estabelecer conexões com o quotidiano e a experiência dos telespectadores (LEÓN, 2008). Assim, convergem a preferência do público, a frequência com que esses temas são abordados e a duração das matérias.

As frequências relativas dos frames "nova pesquisa" e "novo desenvolvimento tecnológico", somadas, perfazem mais da metade das matérias, o que indica que a atualidade de um assunto tem valor alto para o Fantástico, ainda que o programa não se proponha somente a informar. Por outro lado, por sua "dupla natureza" - conjugando informação e entretenimento -, não surpreende que a "personalização" tenha sido frequente no Fantástico, por se tratar de um dos recursos mais comumente utilizados na televisão para criar identificação com suas audiências (KITZINGER, 1999; BRANTS, 1998). A personalização usada pelos programas de infotainment pode ser uma estratégia relevante para o público acomodar as informações e enquadrar os temas sociais em perspectivas pessoais (BRANTS, 1998, 56).

Cientistas, cidadãos comuns, médicos e outros especialistas foram as fontes mais consultadas pelos jornalistas do Fantástico na cobertura de C\&T, diferentemente da cobertura da gripe A/ H1N1 em 2009 (mesmo período do presente estudo), na qual representantes de governo e médicos figuraram como as fontes 
principais tanto no Jornal Nacional, um programa noticioso, como no programa de infotainment (MEDEIROS, MASSARANI, 2011; MEDEIROS, MASSARANI, 2010). Observa-se, portanto, que o dominical brasileiro confere grande peso ao conhecimento de especialistas em Ciências e Saúde, cuja autoridade contribui para a construção de uma imagem de credibilidade pelo programa, mas também recorre com frequência a cidadãos comuns para inscrever a narrativa no quotidiano e tornar os tópicos pessoalmente relevantes para suas audiências (KITZINGER, 1999; HENDERSON, KITZINGER, 1999). Por outro lado, também pode dramatizar os temas confrontando o conhecimento disseminado pelo senso comum com o conhecimento científico (RONDELLI, 2004; RUBBO, 2007).

Em documentários sobre Medicina e Saúde, cidadãos comuns são usados para personalizar e normalizar os cuidados e para legitimar a supervisão e a intervenção médica (HODGETTS, CHAMBERLAIN, 1999). No Brasil, em reportagens veiculadas por telejornais sobre o câncer, cidadãos comuns tiveram maior projeção que os especialistas, estratégia utilizada para aproximar o tema do público. Segundo Jurberg e Verjovsky (2010), a Ciência, quando aparece, é usada como ferramenta de apoio, "para 'respaldar cientificamente' uma informação sobre Saúde que pretende ser transmitida". No Fantástico, por outro lado, a Ciência aparece como explicação, aconselhamento, comprovação e investigação, tendo predominado as duas primeiras formas (RONDELLI, 2004; RUBBO, 2007).

Os resultados obtidos neste estudo mostram ênfase em temas científicos nacionais, em consonância com a tendência observada no Jornal Nacional (RAMALHO, POLINO, MASSARANI, 2012), indicando a valorização da Ciência produzida no país por dois programas do horário nobre da TV Globo. Como ocorre com o Jornal Nacional, Fantástico apresenta uma cobertura de C\&T fortemente concentrada geograficamente, sendo a maior parte das matérias focada no Brasil e nos Estados Unidos; a Ciência de outros países latino-americanos e do mundo em desenvolvimento é praticamente invisível. 
Em contraste com outros estudos sobre a cobertura de C\&T, observamos que explicações científicas foram frequentes no Fantástico - presentes em $60 \%$ das matérias. Nos telejornais europeus, León (2008) encontrou explicações breves e simples em 14,68\% das matérias que analisou. Por outro lado, em consonância com outros estudos sobre C\&T na mídia, benefícios e promessas foram mencionados com maior frequência que danos e riscos no Fantástico. Desse modo, pode-se dizer que o programa se afirma como lócus de reforço, reafirmação e legitimação dos poderes e das promessas da Ciência (SIQUEIRA, 1998; RONDELLI, 2004). Ainda assim, controvérsias foram mencionadas em um terço das matérias. Mas controvérsias não científicas, ou seja, que não dizem respeito a produtos ou processos da Ciência foram mais frequentes que as científicas, nas quais os cientistas são chamados a opinar como especialistas.

O uso de recursos visuais no Fantástico foi muito frequente, podendo ter contribuído não somente para a dramatização dos temas, como também para facilitar a compreensão pelo público, conforme se dá também em documentários e programas sobre $\mathrm{Ci}$ ência. Imagens de cientistas também foram altamente frequentes. Quanto maior o destaque que tiverem, melhor para a Ciência, porque um número maior de pessoas se disporá a ouvir o que os cientistas têm a dizer, o que pode influenciar até o recrutamento, isto é, a atração de jovens estudantes para carreiras científicas.

Cientistas homens apareceram no Fantástico com frequência bem maior que mulheres. Essa é uma imagem estereotípica da Ciência que se mostra cada vez mais distante da realidade no Brasil e no mundo, onde existem áreas científicas nas quais há mais mulheres que homens em atividade. Seria necessária, porém, uma análise qualitativa para examinar se o Fantástico reproduz estereótipos de gênero em suas matérias de Ciência. Quanto ao estereótipo do cientista, geralmente vinculado ao trabalho em laboratórios, manipulando equipamentos estranhos e vidrarias, observamos que esses aspectos não foram tão frequentes, já que os cientistas foram retratados em laboratórios em menos de um terço das matérias. 
Apesar dos problemas que possam ter, programas de entretenimento e infotainment são relevantes para a divulgação da Ciência. No contexto de um museu de Ciência, enquete realizada por telefone em Los Angeles, Califórnia (EUA), verificou que 43,0\% das pessoas relacionavam a maior compreensão da Ciência com o "aprendizado por escolha". Os conhecimentos sobre Ciência eram adquiridos, primeiramente, por interesse pessoal, necessidade e/ ou curiosidade em atividades de lazer (FALK, STORKSDIECK, DIERCKING, 2007). A princípio, o caráter misto do Fantástico não parece afetar negativamente a cobertura de Ciência e Tecnologia, como o infotainment não necessariamente deturpa ou deteriora a cobertura da política, por exemplo (BRANTS, 1998). Pelo contrário: propondo-se a ser uma revista eletrônica, dá lugar para reportagens mais longas que as apresentadas rotineiramente em programas noticiosos. A maior duração das matérias também contribui para a inclusão de elementos cuja ausência ou insufiCiência na cobertura de Ciência é criticada, como a explicação de termos científicos.

No que diz respeito à influência que os meios de Comunicação exercem sobre o público, vários autores mostram que a relação entre o conteúdo veiculado pela mídia e as audiências não é unilateral nem direta (PETERS, 2000; LIU, PRIEST, 2009; PRIEST, GREENHALGH, KRAMER, 2010). Peters (2000), por exemplo, em seu estudo sobre recepção de matérias de jornais e TV sobre Engenharia Genética, observou que a atitude das pessoas após o contato com as matérias correspondia, em grande medida, às suas pré-atitudes e que a orientação positiva ou negativa do material jornalístico sobre o tema não tinha relação direta com a opinião dos participantes após a leitura/observação das notícias. Ainda assim, é inegável a importância dos meios de Comunicação como fonte de informação e como elemento que colabora (juntamente com outros elementos) na construção da percepção pública diante de diversos temas, aqui em particular da Ciência e da Tecnologia. Partindo dos indicadores descritos nesse artigo, resta avaliar mais a fundo a qualidade da cobertura e investigar os possíveis efeitos que exerce sobre suas audiências. 


\section{Referências}

ALBERTSON, Bethany; LAWRENCE, Adria. After the credits roll - the long-term effects of educational television on public knowledge and attitudes. American Politics Research, Thousand Oaks, Sage, v. 37, n. 2, p. 275-300, mar. 2009.

ALDRIDGE, Meryl; DINGWALL, Robert. Teleology on television? Implicit models of evolution in broadcast wildlife and nature programmes. European Journal of Communication, London, Sage, v. 18, n. 4, p. 435-453, dec. 2003.

BATES, Benjamin R. Public culture and public understanding of genetics: a focus group study. Public Understanding of Science, London, Sage, v. 14, n. 1, p. 47-65, jan. 2005.

BESLEY, John C.; SHANAHAN, James. Media attention and exposure in relation to support for agricultural biotechnology. Science Communication, Thousand Oaks, Sage, v. 26, n. 4, p. 347-367, jun. 2005.

BRANTS, Kees. Who is afraid of infotainment? European Journal of Communication, London, Sage, v. 13, n. 3, p. 315-335, Set. 1998.

BUCCHI, Massimiano; MAZZOLINI, R. Big science, little news: science coverage in the Italian daily press, 1946-1997. Public Understanding of Science, London, Sage, v.12, n.1, p.7-24, jan. 2003.

CHAPMAN, Simon; HOLDING, Simon J.; ELLERM, Jessica et al. The content and structure of Australian television reportage on health and medicine, 2005-2009: parameters to guide health workers. Medical Journal of Australia, Strawberry Hills, Australian Medical Association, v. 191, n. 11-12, p. 620-624, dec. 2009.

DINGWALL, Robert; ALDRIDGE, Meryl. Television wildlife documentary programming as a source of popular scientific information: a case study in evolution. Public Understanding of Science, London, Sage, v. 15, n. 2, p. 131-152, abr. 2006.

DURANT, John; EVANS, Geoffrey; THOMAS, Geoffrey. Public understanding of science in Britain: the role of medicine in the popular representation of science. Public Understanding of Science, London, Sage, v.1, n.2, p.161-182, 1992.

EINSIEDEL, Edna. Framing science and technology in the Canadian Press. Public Understanding of Science, London, Sage, v.1, n.1, p.89-101, 1992. 
EUROPEAN COMMISSION. Social values, science and technology (Special Eurobarometer 225, Wave 63.1). Brussels: European Commission, 2005. Disponível em: http://ec.europa.eu/public_opinion/archives/ebs/ebs_225_report_ en.pdf. Acesso em: 27 jan. 2012.

FALK, John H.; STORKSDIECK, Martin; DIERKING, Lynn D. Investigating public science interest and understanding: evidence for the importance of free-choice learning. Public Understanding of Science, London, Sage, v. 16, n. 4, p. 455-469, out. 2007.

GÖRKE, Alexander; RUHRMANN, Georg. Public communication between facts and fictions: on the construction of genetic risk. Public Understanding of Science, London, Sage, v. 12, n. 3, p. 229-241, jul. 2003.

HENDERSON, Leslie; KITZINGER, Jenny. The human drama of genetics: 'hard' and 'soft' media representations of inherited breast cancer. Sociology of Health \& Illness. Oxford, Wiley-Blackwell and the Foundation for the Sociology of Health and Illness, v. 21, n. 5, p. 560-578, 1999.

HODGETTS, Darrin; CHAMBERLAIN, Kerry. Medicalization and the depiction of lay people in television health documentary. Health, London, Sage, v. 3, n. 3, p. 317-333, Jul. 1999.

HOLBERT, R. Lance; KWAK, Nojin; SHAH, Dhavan V. Environmental concern, patterns of television viewing, and pro-environmental behaviors: integrating models of media consumption and effects. Journal of Broadcasting and Electronic Media, New York, Broadcast Education Association, v. 47, n. 2, p. 177-196, 2003.

JÖRG, Daniele. The good, the bad and the ugly - Dr. Moreau goes to Hollywood. Public Understanding of Science, London, Sage, v. 12, n. 3, p. 297-305, jul. 2003.

JURBERG, Claudia; VERJOVSKY, Marina. Nunca aos domingos: um estudo sobre a temática do câncer nas emissoras de TV brasileiras. História, Ciências, Saúde - Manguinhos, Rio de Janeiro, Casa de Oswaldo Cruz/Fundação Oswaldo Cruz, v. 17, supl. 1, p. 145-163, jul. 2010.

KITZINGER, Jenny. Researching risk and the media. Health, Risk \& Society, New York, Routledge, v. 1, n. 1, p. 55-69, 1999.

LEÓN, Bienvenido. Science-related information in European television: a study of prime-time news. Public Understanding of Science, London, Sage, v. 17 , n. 4, p. 443-460, out. 2008. 
LIU, Hui; PRIEST, Susanna. Understanding public support for stem cell research: media communication, interpersonal communication and trust in key actors. Public Understanding of Science, London, Sage, v. 18, n. 6, p. 704-718, nov. 2009

LOWE, Thomas; BROWN, Katrina; DESSAI, Suraje et al. Does tomorrow ever come? Disaster narrative and public perceptions of climate change. $\mathrm{Pu}$ blic Understanding of Science, London, Sage, v. 15, n. 4, p. 435-457, Oct. 2006.

MEDEIROS, Flavia N.S.; MASSARANI, Luisa. A cobertura da gripe A/H1N1 pelo Fantástico. Revista Brasileira de Ciências da Comunicação, São Paulo, Intercom, v. 34, n. 1, p. 41-59, jan./jun. 2011.

MEDEIROS, Flavia N.S.; MASSARANI, Luisa. Pandemia no ar: estudo de caso sobre a cobertura da gripe A/H1N1 por um telejornal brasileiro. JCOM, Trieste, Sissa, v. 9, n. 3, A03, Sep. 2010.

MILLER, Jon D.; AUGENBRAUN, Eliene; SCHULHOF, Julia et al. Adult science learning from local television newscasts. Science Communication, Thousand Oaks, Sage, v. 28, n. 2, p. 216-242, dez. 2006.

NISBET, Matthew C.; SCHEUFELE, Dietram A.; SHANAHAN, James et al. Knowledge, promise or reservations?: a media effects model for public perceptions of science and technology. Communication Research, Thousand Oaks, Sage, v. 29, n. 5, p. 584-608, out. 2002.

NUCCI, Mary L.; KUBEY, Robert. "We begin tonight with fruits and vegetables": genetically modified food on the evening news. Science Communication, Thousand Oaks, Sage, v. 29, n. 2, p. 147-176, dez. 2007.

PADIGLIONE, Cristina. Globo e Record caem, SBT sobe no Ibope 2011, tudo em migalhas. O Estado de S.Paulo, São Paulo, 30 dez. 2011. Disponível em: http://www.estadao.com.br/noticias/arteelazer,globo-e-record-caem-sbt-sobe-no-ibope-2011-tudo-em-migalhas,816784,0.htm. Acesso em 12 jan. 2012.

PRIEST, Susanna; GREENHALGH, Ted; KRAMER, Victoria. Risk perceptions starting to shift? U.S. citizens are forming opinions about nanotechnology. Journal of Nanoparticle Research, New York, Springer, v.12, n.1, p.11-20, 2010.

PETERS, Hans Peter. The committed are hard to persuade. Recipients' thoughts during exposure to newspaper and TV stories on genetic engineering and their effect on attitudes. New Genetics and Society, London, Routledge, v. 19, n. 3, p. 365-381, 2000. 
RAMALHO, Marina, POLINO, Carmelo, MASSARANI, Luisa. Do laboratório para o horário nobre: a cobertura de Ciência no principal telejornal brasileiro. JCOM, Trieste, Sissa, v.11, n.2, p. A02, Jun.2012. Disponível em: http:// jcom.sissa.it/archive/11/02/Jcom1102(2012)A02/Jcom1102(2012)A02_po.pdf. Acesso em 20 jul. 2012.

RONDELLI, Daniela R.R. A Ciência no picadeiro: uma análise das reportagens sobre Ciência no programa Fantástico. 2004, 147 f. Dissertação (Mestrado em Comunicação Social) - Programa de Pós-Graduação em Comunicação Social, Universidade Metodista de São Paulo.

RUBBO, Daniela. A Ciência no Fantástico: uma análise de discurso. In: CONGRESSO BRASILEIRO DE CIÊNCIAS DA COMUNICAÇÃO. Santos, 2 set. 2007.

SIQUEIRA, Denise. Ciência na televisão: mito, ritual e espetáculo. Revista Brasileira de Ciências da Comunicação, São Paulo, Intercom, v.21, n.2, p.57-69, jul-dez. 1998.

SURETTE, Ray; OTTO, Charles. A test of crime and justice infotainment measure. Journal of Criminal Justice, Amsterdam:,Elsevier, v. 30, n. 5, p. 443-453, set./out. 2002.

VERHOEVEN, Piet. Sound-bite science: on the brevity of science and scientific experts in Western European television news. Science Communication, Thousand Oaks, Sage, v. 32, n. 3, p. 330-355, Sep. 2010.

VeTteHEN, Paul H.; NUIJTEN, Koos; BEENTJES, Johannes W.J. Sensationalism in Dutch current affairs programmes 1992-2001. European Journal of Communication, London, Sage, v. 21, n. 2, p. 227-237, Jun. 2006.

WEINGART, Peter; MUHL, Claudia; PANSEGRAU, Petra. Of power maniacs and unethical geniuses: science and scientists in fiction film. Public Understanding of Science, London, Sage, v. 12, n. 3, p. 279-287, Jul. 2003.

Obs.: As atribuições dos autores neste artigo são como a seguir: Natércia teve um papel protagonista na elaboração do texto deste artigo. Caldas e Ramalho participaram da elaboração deste texto e da codificação dos dados, sendo que a última participou ativamente do grupo que concebeu os protocolos utilizados em nosso estudo e capacitou os codificadores associados ao projeto. Massarani coordena o grupo e participou ativamente de todas as etapas, desde a construção dos protocolos até a elaboração do texto deste artigo.

Recebido: 24/10/2012 Aceito: 05/04/2013 